The Astrophysical Journal, 689:1274-1278, 2008 December 20

(C) 2008. The American Astronomical Society. All rights reserved. Printed in U.S.A.

\title{
BINARITY IN COOL ASYMPTOTIC GIANT BRANCH STARS: A GALEX SEARCH FOR ULTRAVIOLET EXCESSES
}

\author{
R. Sahai, ${ }^{1}$ K. Findeisen, ${ }^{2}$ A. Gil de Paz, ${ }^{3}$ and C. Sánchez Contreras ${ }^{4}$ \\ Received 2008 April 22; accepted 2008 August 12
}

\begin{abstract}
The search for binarity in AGB stars is of critical importance for our understanding of how planetary nebulae acquire the dazzling variety of aspherical shapes which characterizes this class. However, detecting binary companions in such stars has been severely hampered due to their extreme luminosities and pulsations. We have carried out a small imaging survey of AGB stars in ultraviolet light (using GALEX), where these cool objects are very faint, in order to search for hotter companions. We report the discovery of significant far-ultraviolet excesses toward nine of these stars. The far-ultraviolet excess most likely results either directly from the presence of a hot binary companion or indirectly from a hot accretion disk around the companion.
\end{abstract}

Subject headings: binaries: general — circumstellar matter — planetary nebulae: general stars: AGB and post-AGB - stars: mass loss

\section{INTRODUCTION}

There are many observational indications which lead us to believe that binarity, believed to be very common among pre-mainsequence (e.g., Bodenheimer et al. 2000) and main-sequence stars (Duquennoy \& Mayor 1991), strongly influences the history and geometry of mass loss during the late stages of stellar evolution. The evolutionary transition from the AGB to the post-AGB phase is accompanied by significant changes in the morphology of these objects - the roughly round circumstellar mass-loss envelopes (CSEs) of AGB stars evolve into post-AGB nebulae with a dazzling variety of shapes and intriguing symmetries (e.g., Schwarz et al. 1992; Sahai \& Trauger 1998; Sahai et al. 2007). Critical reviews (Soker 1998) of the properties of bipolar PNe (e.g., Corradi \& Schwarz 1995) lead to the conclusion that binary models can explain all these properties, whereas single-star models (e.g., GarcíaSegura 1997) have many difficulties.

However, in spite of dedicated efforts by many researchers to search for binarity in evolved stars, direct observational evidence for binarity has been hard to come by. AGB stars are very luminous $\left(\sim\right.$ few $\left.\times 10^{3}-10^{4} L_{\odot}\right)$ and surrounded by dusty envelopes, making it very difficult to directly detect nearby stellar companions, which are generally likely to be significantly less luminous main-sequence stars or white dwarfs. Indirect techniques such as radial velocity measurements (e.g., van Winckel et al. 1999; Sorensen \& Pollacco 2003; De Marco et al. 2004) or photometric variability measurements (Bond 2000) have been used for the central stars of PNs and post-AGB objects, with some success. But these techniques cannot be easily applied to AGB stars, because the latter show strong variability intrinsic to their pulsating atmospheres, which potentially masks the corresponding variability due to a companion. Extensive observations of the central stars of planetary nebulae have resulted in detections a sum total of $\lesssim 20$ binaries (Bond 2000; Ciardullo et al. 1999), implying a $10 \%-15 \%$ fraction of detectable close binaries among randomly selected PNe. Bond (2000) concludes that it is likely that the

\footnotetext{
1 Jet Propulsion Laboratory, Mail Stop 183-900, California Institute of Technology, Pasadena, CA 91109.

2 Cornell University, Ithaca, NY 14853.

3 Departamento de Astrofísica, Universidad Complutense de Madrid, 28040 Madrid, Spain.

4 Departamento de Astrofísica Molecular e Infraroja, Instituto de Estructura de la Materia-CSIC, Serrano 121, 28006 Madrid, Spain.
}

known short-period binaries in PNe are only the tip of an iceberg of a substantial population of longer period binaries.

Deep ultraviolet observations hold the promise of allowing us to discover substantial numbers of binary companions in AGB stars, since most mass-losing AGB stars are relatively cool objects (spectral types $\sim \mathrm{M} 6$ or later). The companions are likely to be main-sequence stars because of the steep dependence of evolutionary rates on stellar mass (e.g., Soker \& Rappaport 2001). Thus, for a secondary-to-primary mass ratio, $q=M_{2} / M_{1}$, around unity, any stellar companion has a good probability of being hotter than the primary. However, it is difficult to estimate with confidence the number of such systems in which the secondary is on the mainsequence and hotter than the primary, as a fraction of the total number of primordial binaries, since the mass-ratio probability distribution, $f(q)$, where $q=M_{2} / M_{1}$, is not well known. A promising approach is to carry out population synthesis studies (which are still in their infancy) such as those of Soker \& Rappaport (2000), who adopt for their modeling $f(q) \propto q^{1 / 4}$; note that this function is not strongly peaked toward $q=1$.

Since observed and model spectra of cool AGB stars show that their fluxes die rapidly at wavelengths shortward of about $2800 \AA$, significantly favorable secondary-to-primary flux contrast ratios $(>10)$ for companion detection may be reached in the GALEX FUV (1344-1786 ̊) and NUV (1771-2831 ̊̊) bands, for companions of spectral type hotter than about $\mathrm{G0}\left(T_{\text {eff }}=6000 \mathrm{~K}\right)$. In this paper we report on a subsample of objects from our cycle 1 pilot program which were detected in both the FUV and NUV bands and on the implications of these detections for binarity. A comprehensive study covering the full results of our survey will be presented in a forthcoming paper.

\section{OBSERVATIONS AND RESULTS}

We selected a sample of 25 AGB late-M (i.e., M5 or later) stars (which passed the GALEX mission "bright-star" and "highbackground" tests) largely based on their inclusion in the Hipparcos astrometric catalog, with a "multiplicity" flag in the header field H59 of the main catalog, indicating that a single-star astrometric solution was not adequate. ${ }^{5}$ Thus, for $20 / 25$ objects, the selection criteria of our "pilot" program were intentionally biased toward

\footnotetext{
5 These were the so-called problem stars for which a double star solution with the classical parameters of separation and position angle could not be found: and were flagged as $\mathrm{G}, \mathrm{O}, \mathrm{V}$, or X in field $\mathrm{H} 59$ of the main catalog.
} 
optimizing the a priori probability offinding companions, in order to test the validity of our technique. Three objects did not have a "multiplicity" flag; and two are not in the Hipparcos catalog, but were selected from published lists of AGB stars with molecular envelopes detected in $\mathrm{CO}$ emission. Although most of our objects had positive entries for the annual parallax, the errors were usually large, and only for four objects were the parallax measurements significant (i.e., greater than $3 \sigma$ ). The requirement that our objects be M5 (or later) or cool N-type carbon stars was included in order to min0imize the ratio of their UV fluxes to that of hotter companions (if present).

From our original list of 25 objects, 21 objects have been observed - 20 as part of our GI program (GI1-23; PI: R. Sahai) and 1 as part of other programs - in both the NUV (1771-2831 $)$ and FUV (1344-1786 $\AA$ ) bands. Nine of these were detected in both the FUV and NUV bands with high S/N $(\gtrsim 8 \sigma)$ (Table 1). Among these, the NUV image of AF Peg has an elliptical shape, and an intensity cut along the major axis (P.A. $\sim 75^{\circ}$ ) shows two peaks separated by about $6^{\prime \prime}$; the stronger one corresponds to AF Peg's location; in the FUV image, AF Peg is weaker. Because the separation is comparable to the PSF, the two sources cannot be deconvolved reliably; hence, the measured fluxes of AF Peg are very uncertain. We have used the pipeline-generated catalogs included with the imaging data sets for extracting the photometry for the remaining eight sources. Stars not detected in the FUV will be discussed, together with their NUV properties, in a forthcoming paper.

The eight FUV sources include four oxygen-rich stars (RW Boo, AA Cam, V Eri, and R UMa) and four carbon-rich stars (T Dra, TW Hor, V Hya, and VY UMa). For those objects for which more than one exposure taken at different epochs was available, we list both the individual and average fluxes.

The typical uncertainty in the measured fluxes is dominated by systematic uncertainties in the GALEX pipeline photometric calibration of about 10\%-15\% (Morrissey et al. 2005).

We now consider whether our FUV and/or NUV detections could result from the presence of a small-filter red leak, which could produce spurious detection of a UV signal for the extremely red stars observed in this study. Since the GALEX detectors are photon-counting MAMA detectors, there is supposedly no red leak, since only UV photons can trigger the photoelectrons (Rich 2005); the photocathode on the FUV detector is nonresponsive above $\sim 1800 \AA$, and the NUV response is suppressed below measurable levels by multilayer coatings on the optics. According to the GALEX help desk, there is no measurable red leak in either GALEX band.

Even though no red leak response has been measured for the GALEX filters, we have ensured that even if such a response is present at a low level, our modeling is not affected because the upper limits that we can set on the red leak from our data are quite low. We have done this by comparing the ratios of the FUV and NUV fluxes to the $V$-band fluxes in our survey objects and assuming that the lowest of these ratios is due to a red leak. From this analysis, we find values of $2.5 \times 10^{-7}$ and $4 \times 10^{-6}$ for the maximum possible red leak flux in the GALEX FUV and NUV bands, as a fraction of the $V$-band flux - and these ratios are too low to affect our models or the detection statistics we report in this paper.

\section{ULTRAVIOLET EXCESSES}

We now investigate the origin of the ultraviolet fluxes in the objects we have detected in the FUV band. We have fitted the spectral energy distributions (SEDs) from 0.1 to $2 \mu \mathrm{m}$ (Fig. 1) of the four oxygen-rich stars with reliable FUV fluxes (i.e., RW Boo, AA Cam, V Eri, and R UMa), using stellar atmosphere models of AGB stars (Fluks et al. 1994), corresponding to the spectral type of each star as given in the General Catalogue of Variable Stars
TABLE 1

AGB Stars with UV Excesses

\begin{tabular}{|c|c|c|c|c|}
\hline Target & Band & Epoch $^{\mathrm{a}}$ & $\begin{array}{l}\text { Exposure Time } \\
\text { (s) }\end{array}$ & $\begin{array}{l}\text { Flux } \\
\text { (mJy) }\end{array}$ \\
\hline \multirow[t]{4}{*}{ RW Boo ${ }^{\mathrm{b}} \ldots \ldots \ldots \ldots \ldots \ldots$} & FUV & 4220.75 & 1726 & 0.026 \\
\hline & NUV & 3861.15 & 3396 & 0.47 \\
\hline & & 4220.75 & 1726 & 0.39 \\
\hline & & Average & 5122 & 0.44 \\
\hline \multirow[t]{4}{*}{ AA Cam } & FUV & 3377.48 & 1693 & 0.014 \\
\hline & NUV & 3377.48 & 1532 & 0.22 \\
\hline & & 3425.29 & 1693 & 0.28 \\
\hline & & Average & 3225 & 0.25 \\
\hline \multirow[t]{5}{*}{ 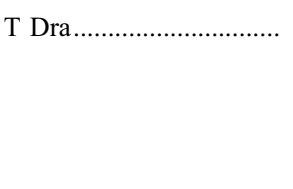 } & FUV & 3984.2 & 1580 & 0.0055 \\
\hline & NUV & 3541.9 & 1250 & 0.017 \\
\hline & & 3587.5 & 1309 & 0.018 \\
\hline & & 3984.2 & 1580 & 0.032 \\
\hline & & Average & 4151 & 0.021 \\
\hline \multirow[t]{2}{*}{ V Eri } & FUV & 3678.20 & 1620 & 0.060 \\
\hline & NUV & 3678.20 & 1704 & 0.14 \\
\hline \multirow[t]{15}{*}{ 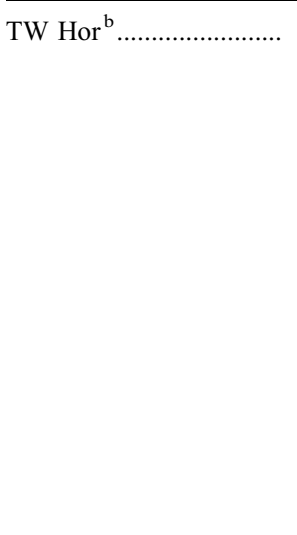 } & FUV & 3349.3 & 4381 & 0.026 \\
\hline & & 3351.2 & 1345 & 0.032 \\
\hline & & 3671.8 & 109 & 0.034 \\
\hline & & 3706.6 & 255 & 0.023 \\
\hline & & 3706.7 & 586 & 0.033 \\
\hline & & 4018.8 & 747 & 0.034 \\
\hline & & Average & 7423 & 0.027 \\
\hline & NUV & 3349.3 & 4381 & 0.53 \\
\hline & & 3351.2 & 1345 & 0.55 \\
\hline & & 3359.25 & 2214 & 0.34 \\
\hline & & 3671.8 & 109 & 0.78 \\
\hline & & 3706.6 & 339 & 0.26 \\
\hline & & 3706.7 & 645 & 0.27 \\
\hline & & 4018.8 & 747 & 0.99 \\
\hline & & Average & 9780 & 0.49 \\
\hline \multirow[t]{6}{*}{ 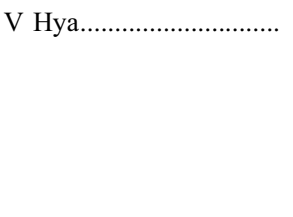 } & FUV & 3421.86 & 1705 & 0.12 \\
\hline & & 3778.54 & 1003 & 0.15 \\
\hline & & Average & 2708 & 0.13 \\
\hline & NUV & 3421.86 & 1705 & 0.11 \\
\hline & & 3778.54 & 1003 & 0.13 \\
\hline & & Average & 2708 & 0.12 \\
\hline \multirow[t]{2}{*}{ AF Peg ${ }^{\mathrm{c}}$} & FUV & 3280.70 & 1551 & 0.011 \\
\hline & NUV & 3280.70 & 1551 & 0.090 \\
\hline \multirow[t]{2}{*}{ R UMa } & FUV & 3742.14 & 1703 & 0.041 \\
\hline & NUV & 3742.14 & 1703 & 0.12 \\
\hline \multirow[t]{2}{*}{ 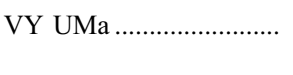 } & FUV & 3742.50 & 1704 & 0.0061 \\
\hline & NUV & 3742.50 & 1704 & 0.23 \\
\hline
\end{tabular}

a JD $-2,450,000$.

b Observed at multiple epochs; data from epochs separated by $\lesssim 0.1$ day are averaged together in the individual-epoch rows.

${ }^{c}$ Observed at two epochs in the NUV band only; quoted fluxes are for AF Peg using a two-Gaussian fit to the image of the latter and the partially blended nearby star, for epoch 1.

(GCVS). ${ }^{6}$ A visual extinction, $A_{V}$, to account for the extinction by circumstellar dust due to the dusty mass-loss envelope of the primary, is also determined from our fits. Archival photometry at wavelengths redward of the GALEX NUV band was taken from the Hubble Guide Star Catalog (GSC 2.2), the US Naval Observatory USNO-B1.0 Catalog, and the Two Micron All-Sky Survey.

\footnotetext{
${ }^{6}$ Vizier Online Data Catalog, 2250, 0 (N. Samus et al., 2004).
} 

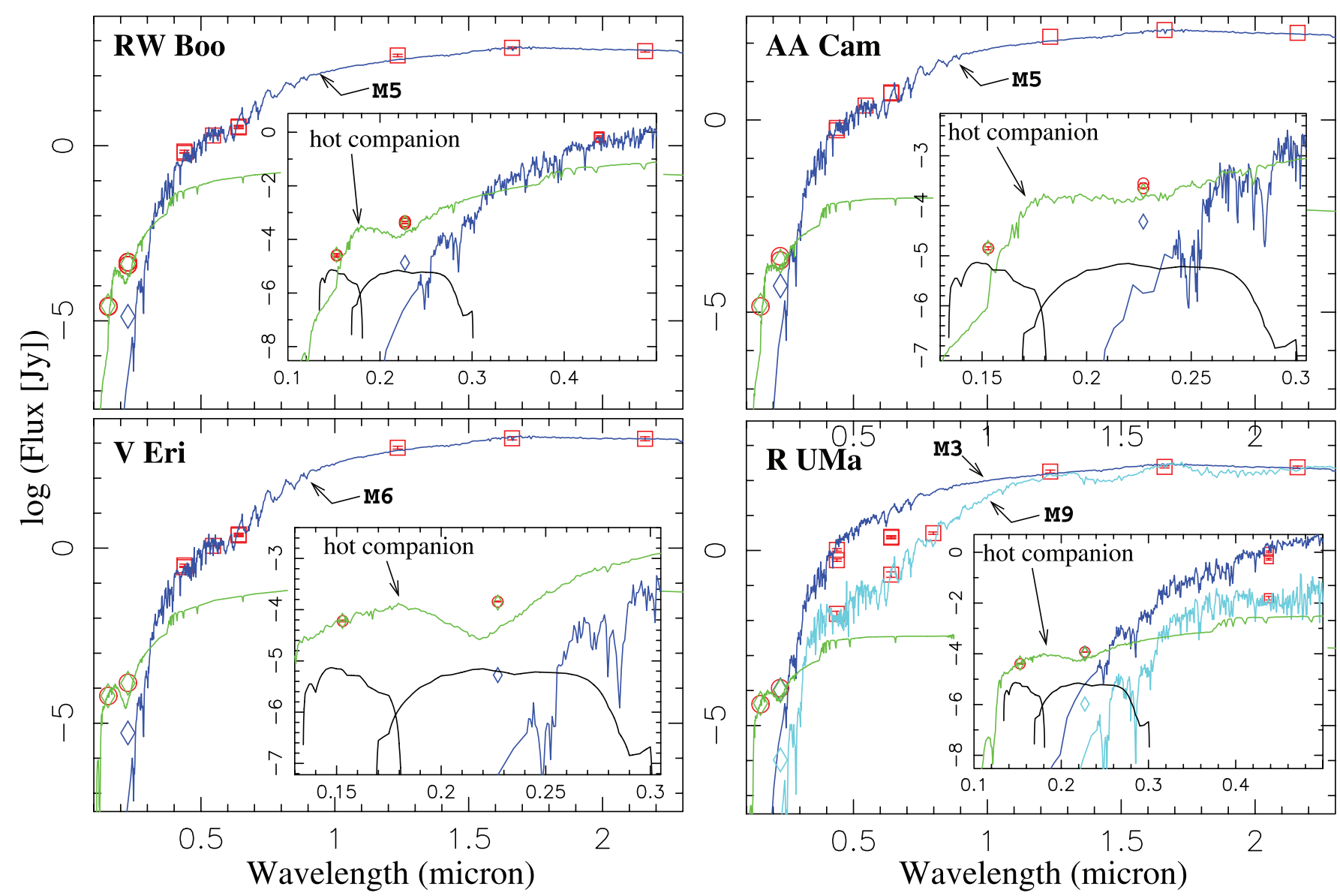

FIG. 1.-GALEX (NUV and FUV) and ground-based (optical and near-IR) fluxes (red symbols) of AA Cam, V Eri, and R UMa, with model spectra (blue: cool AGB star; green: hot companion). The expected NUV flux (blue/cyan diamonds) from the cool AGB star (the expected FUV flux due to AGB star lies below the minimum of the flux range), and from the hot companion+cool AGB star (green diamonds) are also shown. The inset shows an expanded view of the UV-blue region. The model NUV/FUV fluxes (diamonds) are obtained by convolving the model spectrum with the GALEX NUV and FUV bandpasses (black curves, insets).

We find that the observed FUV (NUV) fluxes are a factor $>10^{6}(>5)$ larger than expected for the photospheric emission of the primary, accounting for the finite filter bandwidth, the filter response, ${ }^{7}$ and the steeply sloping spectrum in the UV. Hence, even though the photometric variability of the primary stars makes our fit somewhat uncertain, it certainly cannot account for the FUV excesses because they are very large. Moreover, there is no systematic relationship between the light-cycle phases of the various photometric data points used to fit the primary model which could conspire to produce such an excess in each of our three sources.

The detailed SED fitting described could not be carried out for the four carbon-rich stars in Table 1 because for these objects model atmospheres for $\lambda \lesssim 2300 \AA$ are not available (D. Luttermoser, private communication). We therefore used blackbody spectra based on $T_{\text {eff }}$ values from Bergeat et al. (2001) and scaled these to fit the NIR and optical photometry of each object. We find that even for the object with the smallest FUV excess (VY UMa) the model FUV flux of the primary AGB star is lower than the observed value by a factor $\sim 30$. V Hya stands out among the nine FUV-detected stars as having the highest FUV-to-NUV flux ratio $(\gtrsim 1)$, but the coolest photosphere $\left(T_{\text {eff }}=2160 \mathrm{~K}\right)$ for the primary. We discuss our detection of the FUV/NUV excess in this object in more detail in $\S 4$.

We note that Wood \& Karovska (2004), based on International Ultraviolet Explorer (IUE) spectroscopic data of several Mira AGB

\footnotetext{
${ }^{7}$ Taken from http://galexgi.gsfc.nasa.gov/docs/galex/tools/Resolution_Response/ index.html.
}

stars at multiple phases, conclude that this class of objects does not produce any detectable emission below $2000 \AA$. We have examined the IUE database for the sources in our survey and find that only three objects in our survey sample were observed: R LMi, V Hya, and TW Hor. R LMi and TW Hor were observed with both the long-wavelength (LWR and LWP) and short-wavelength (SWP) instruments (Boggess et al. 1978a, 1978b), whereas V Hya was observed only with the long-wavelength instrument. For both $\mathrm{R}$ Lmi and V Hya, the signal-to-noise ratio $(\mathrm{S} / \mathrm{N})$ over the observed bandpasses (1910-3300 ̊ for LWR/LWP and 1150$1975 \AA$ for the SWP) is close to zero. For TW Hor the situation is similar except at wavelengths longer than $\sim 2500 \AA$, where significant flux is detected; the steady rise in the spectrum toward the red end of the bandpass indicates that this flux is most likely due to the primary star. We have convolved the IUE spectra with the GALEX FUV and NUV filter bandpasses in order to compute the GALEX-equivalent fluxes (or upper limits) for these sources. For R LMi we find $3 \sigma$ upper limits of $0.032 \mathrm{mJy}$ (FUV) and $0.16 \mathrm{mJy}$ (NUV). For the other two sources, the derived fluxes ( $3 \sigma$ errors) are $0.096 \pm 0.051 \mathrm{mJy}$ (FUV) and $0.41 \pm 0.05 \mathrm{mJy}$ (NUV) for TW Hor, and $0.12 \pm 0.11 \mathrm{mJy}$ (NUV) for V Hya.

We now examine two plausible explanations for the FUV excesses, both of which involve the presence of a companion star.

\subsection{A Hot Companion}

The NUV and FUV excesses may result from the presence of a companion star which is significantly hotter than the primary. We have therefore made least-squares fits to the FUV and NUV 
TABLE 2

Model Results

\begin{tabular}{|c|c|c|c|c|c|c|}
\hline Target & Primary Spectral Type & $\begin{array}{c}D \\
(\mathrm{kpc})\end{array}$ & $A_{V}$ & $\begin{array}{l}T_{\text {eff }}{ }^{\mathrm{a}} \\
(\mathrm{K})\end{array}$ & $\begin{array}{c}L_{c}^{\mathrm{a}} \\
\left(L_{\odot}\right)\end{array}$ & $L_{p} / L_{c}$ \\
\hline RW Boo ............................ & M5 & 0.32 & 2.3 & $8200(-500,300)$ & $18(-5,7)$ & $280(-80,110)$ \\
\hline AA Cam........................... & M5 & 0.5 & 1.0 & $8200(-400,400)$ & $1.1(-0.6,0.7)$ & $3200(-1300,4500)$ \\
\hline V Eri …….......................... & M6 & 0.22 & 2.9 & $10,000(-700,4100)$ & $6.2(-2.9,2.2)$ & $910(-240,810)$ \\
\hline R UMa ……...................... & M3-M9 & 0.5 & 1.3 & $9200(-300,1100)$ & $0.85(0.2,-0.4)$ & $5300(-900,4700)$ \\
\hline
\end{tabular}

a The numbers in parenthesis represent $3 \sigma$ modeling uncertainties; temperature and luminosity uncertainties are inversely correlated.

excesses of each object by including the contribution of a companion star ( Table 2). We have used models by Kurucz (Castelli \& Kurucz 2003) for the companion spectra.

For RW Boo, AA Cam, and V Eri, the same value of $A_{V}$ was applied to the model spectrum of the companion, as derived from fitting the primary. In the case of R UMa, which is listed in GCVS with a spectral type $\mathrm{M} 3-\mathrm{M} 9$, the best fits to the optical and nearinfrared fluxes were obtained with $A_{V}=2.6(0.0)$ for an M3 (M9) primary spectrum. Therefore, in our least-squares fitting of the companion, we tried three values of $A_{V}(0,1.3$, and 2.6) to scale the companion blackbody spectrum.

Our modeling (Table 2) provides the fractional luminosity (relative to the primary) of the companion. For RW Boo and V Eri, the Hipparcos parallaxes, $3.09 \pm 1.1$ and $4.56 \pm 1.08$ mas, give distances of 320 and $220 \mathrm{pc}$, respectively, implying companion luminosities of $L_{c}=18$ and $6 L_{\odot}$ for these two sources. For AA Cam and R UMa, the parallax measurements are not significant, and the luminosities given in Table 2 are for a nominal distance of $0.5 \mathrm{kpc}$.

The value of $L_{c}$ for RW Boo is consistent with that expected for a mid-A main-sequence star, but it is too low in the case of AA Cam, R UMa, and V Eri, since the luminosities of mainsequence stars using our most favorable (i.e., lowest) model values for each of these, $T_{\text {eff }}=7800,8900,9250 \mathrm{~K}$ (i.e., spectral type $\sim \mathrm{A} 6$ to $\sim \mathrm{A} 1-\mathrm{A} 2)$, lie in the range $\sim(10-35) L_{\odot}(\mathrm{Cox} 2000$, Table 15.7). An appeal to distance ambiguities for these three stars does not help to resolve the problem of the derived $L_{c}$ values being too low for main-sequence stars. We have tried increasing the source distance in order to bring up $L_{c}$ to its main-sequence values. For AA Cam and R UMa, this exercise results in $L_{p}=$ $2.4 \times 10^{4}$ and $9.3 \times 10^{4} L_{\odot}$, using the most favorable models values in Table 2 -i.e., the lowest values of $T_{\text {eff }}(7800$ and $8900 \mathrm{~K})$ and the highest values of $L_{c}\left(1.79\right.$ and $\left.1.01 L_{\odot}\right)$. The value of $L_{p}$ for R UMa (AA Cam) is certainly (probably) too high for an AGB star. For V Eri, a factor 2.4 increase in the distance is needed to scale up $L_{c}$ to a main-sequence luminosity, but only a factor 1.3 increase is allowed by the uncertainty in its parallax data (by a factor of 1.3). We rule out the possibility that the companions are low-luminosity white dwarfs (WDs) on cooling tracks, because stellar evolutionary models show that by the time WDs have cooled to $10^{4} \mathrm{~K}$, their luminosities are orders of magnitude below $1 L_{\odot}$.
In our models we adopted the extinction curve as tabulated by Whittet (1992). Our quoted modeling uncertainties do not take into account uncertainties in the extinction curve at NUV and FUV wavelengths. We repeated the modeling using extinction curves for the LMC supershell and the SMC bar (Gordon et al. 2003), which along with the Galactic extinction roughly cover the range of curves found in circumstellar dust and are well studied. The results are shown in Table 3. Although the best-fit temperatures all shifted up when the LMC and SMC extinction curves were used, the cooler companion models (i.e., for RW Boo and AA Cam) proved reasonably insensitive to the choice of extinction curve, while dramatic differences were found for R UMa and V Eri. In general, we could not obtain a good fit even with the highest temperature models available $(39,000 \mathrm{~K})$ while using the LMC or SMC curves for these two sources.

\subsection{Accretion onto a Companion Star}

Five out of nine objects in Table 1 were observed on more than one epoch in one or both of the GALEX bands - in each instance, significant photometric variability was observed (Table 1). We have checked that this variability is not due to systematic calibration uncertainties because the average and median fractional differences of the fluxes for the brightest 40 field objects in the images from the different epochs are negligible.

A plausible interpretation for the photometric variability is related to the presence of a nearby companion. This interpretation is motivated by ultraviolet observations of Mira, a symbiotic star in which Mira A is the AGB primary and Mira B is a compact companion (at a separation of $0.6^{\prime \prime}$ ) which is accreting matter from Mira A's wind. IUE spectra of Mira in the wavelength region covered by the GALEX bands show the presence of strong emission lines ascribed to Mira B (Reimers \& Cassatella 1985). The strongest of these (due to O, N, and C, seen by IUE during 19791995 ) were found to fade by a factor $>20$ by $1999-2001$ and then start increasing back to their original levels by 2004 (Wood \& Karovska 2006). Assuming a distance of 107 pc to Mira as recomputed by Knapp et al. (2003) from the Hipparcos Intermediate Astrometric Data using improved astrometric fits and chromaticity corrections, the combined maximum fluxes of such emission lines, if present in our sources, would correspond to an artificial continuum in GALEX's broadband FUV filter of about $0.1 \mathrm{mJy}$ at a

TABLE 3

Best-Fit Temperatures (in Kelvins) under Different Extinction Curves

\begin{tabular}{|c|c|c|c|c|}
\hline Target & $A_{V}$ & Galactic $^{\mathrm{a}}$ & $\mathrm{LMC}^{\mathrm{a}}$ & $\mathrm{SMC}^{\mathrm{a}}$ \\
\hline RW Boo.. & 2.3 & $8200(-500,300)$ & $8700(-500,400)$ & No fit \\
\hline AA Cam & 1.0 & $8200(-400,400)$ & $8500(-400,500)$ & $9000(-400,1400)$ \\
\hline 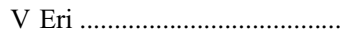 & 2.9 & $10,000(-700,4100)$ & No fit & No fit \\
\hline 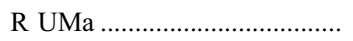 & 1.3 & $9200(-300,1100)$ & $33,000(-22,000)^{\mathrm{b}}$ & No fit \\
\hline
\end{tabular}

${ }^{\text {a }}$ The numbers in parenthesis represent $3 \sigma$ modeling uncertainties.

b The $3 \sigma$ upper limit for R UMa fell beyond the range of available models. 
typical source distance of $350 \mathrm{pc}$, thus comparable to the measured values of the FUV fluxes in our sources. During the IUE era (1979-1980 and 1990-1995), the FUV lines and continuum varied by a factor $\sim 2$ in Mira. Karovska, Wood, and co-authors (Wood et al. 2001, 2002) conclude that the UV variability most likely results from variations in the accretion rate onto Mira B. Although Mira's variability has been observed on a much longer timescale than the ones sampled in our GALEX data, accretion of matter from the primary AGB wind onto a companion provides a plausible explanation for the presence of FUV emission and its variability in our sources.

\section{DISCUSSION}

Our small survey of 21 AGB stars for UV excesses has resulted in a substantial number of NUV and/or FUV detections. Nine of these were detected in the FUV band and are the subject of this paper. A detectable FUV flux at a level of even a few microjanskys is several orders of magnitude too high to be explained by photospheric emission from the relatively cool primary stars in our sample, and hence is an "excess" which requires an alternative explanation - most likely the presence of a binary companion. The excesses arise either as a result of photospheric emission from a hotter companion, and/or from an accretion disk around the companion. Spectroscopic monitoring in the FUV of these sources is needed in order to distinguish between these two mechanisms.

We detected NUV fluxes in 19/21 of our objects with high S/N, many of which are also likely to be "excesses," but for which such an inference is more uncertain because of the significantly larger contribution of the primary in the NUV compared to the FUV. A discussion of the detection statistics of, and the biases in, our full sample is deferred to a forthcoming paper.

Although our discovery of a UV excess attributable to a different star than the primary AGB star does not directly imply that the former is a gravitationally bound companion, it is the most likely explanation. This is because the UV sky is rather "empty" (i.e., much more scarcely populated than at optical wavelengths), and hence the probability, $p_{\text {false }}$, that the FUV-emitting object is simply positionally coincident on the sky with the primary (i.e., lying within a radius of $2^{\prime \prime}$ from the primary) is very small. Using the object number count ( per deg ${ }^{2} \mathrm{mag}$ ) versus magnitude plot for the GALEX FUV band (Bianchi et al. 2007), we find that for AA Cam (the faintest of our modeled sources), $p_{\text {false }}$ for an object of FUV magnitude lying within a $0.5 \mathrm{mag}$ bin centered around the FUV mag of AA Cam, is $8 \times 10^{-5}$. The Galactic latitudes of our objects are similar to those of the fields used by Bianchi et al.; hence, it is appropriate to use their point-source densities.

V Hya, which has the largest FUV flux as well as the highest FUV-to-NUV flux ratio among all our targets, is well known for its collimated, high-velocity, outflows, an extended dusty torus, and an inner hot disk. The outflows were first seen via infrared absorption lines in the $\mathrm{CO} 4.6 \mu \mathrm{m}$ vibration-rotation band (Sahai \& Wannier 1988); recent interferometric mapping of the millimeterwave $\mathrm{CO}$ line emission shows the collimated structure of the fast outflow (e.g., Hirano et al. 2004). More recently, observations with the Hubble Space Telescope (HST) Space Telescope Imaging Spectrograph (STIS) reveal the presence of a high-velocity blob moving away from the central source at (projected) speeds up to $220 \mathrm{~km} \mathrm{~s}^{-1}$ and a hot, slowly expanding $\left(10-15 \mathrm{~km} \mathrm{~s}^{-1}\right)$ central disklike structure (Sahai et al. 2003). Although the expansive kinematics of the latter implies that it is not an accretion disk, the structure may result from a recent phase of equatorially enhanced mass loss, which may be enhancing the accretion process. V Hya is thus the best example to date of an evolved star with an active, collimated outflow, dense equatorially flattened structures possibly related to a central accretion disk, and an inferred binary companion from our UV excess measurements.

We would like to thank an anonymous referee for his/her thoughtful review of our paper. We acknowledge discussions with Patrick Morrissey related to the possibility of red leaks in the GALEX FUV and NUV bands. R. S.'s contribution to the research described in this publication was carried out at the Jet Propulsion Laboratory, California Institute of Technology, under a contract with NASA. R. S. thanks NASA for financial support via a GALEX award and an LTSA award. K. F. was partially funded by a SURF scholarship and through the Cornell Presidential Research Scholars (CPRS) program. A. G. d. P. is partially financed by the Spanish Ramón y Cajal program and the Programa Nacional de Astronomía y Astrofísica under grant AYA 2006-02358. C. S. C. is partially funded for this work by the Spanish MCyT under project AYA 2006-14876 and the Spanish MEC under project PIE 2007501028.
Bergeat, J., Knapik, A., \& Rutily, B. 2001, A\&A, 369, 178

Bianchi, L., et al. 2007, ApJS, 173, 659

Bodenheimer, P., Burkert, A., Klein, R. I., \& Boss, A. P. 2000, in Protostars and Planets IV, ed. V. Mannings, A. P. Boss, and S. S. Russell (Tucson: Univ. Arizona Press), 675

Boggess, A., et al. 1978a, Nature, 275, 372 1978b, Nature, 275, 377

Bond, H. E. 2000, in ASP Conf. Ser. 199, Asymmetrical Planetary Nebulae II: From Origins to Microstructures, ed. J. H. Kastner, N. Soker, \& S. Rappaport (San Francisco: ASP), 115

Castelli, F., \& Kurucz, R. L. 2003, in IAU Symp. 210, Modelling of Stellar Atmospheres, ed. W. W. Piskunov \& D. F. Gray (San Francisco: ASP), A20

Ciardullo, R., Bond, H. E., Sipior, M. S., Fullton, L. K., Zhang, C.-Y., \& Schaefer, K. G. 1999, AJ, 118, 488

Corradi, R. L. M., \& Schwarz, H. E. 1995, A\&A, 293, 871

Cox, A. N. 2000, Allen's Astrophysical Quantities (4th ed.; New York: Springer) De Marco, O., Bond, H. E., Harmer, D., \& Fleming, A. J. 2004, ApJ, 602, L93

Duquennoy, A., \& Mayor, M. 1991, A\&A, 248, 485

Fluks, M. A., Plez, B., The, P. S., de Winter, D., Westerlund, B. E., \& Steenman, H. C. 1994, A\&AS, 105, 311

Garcia-Segura, G. 1997, ApJ, 489, L189

Gordon, K. D., Clayton, G. C., Misselt, K. A., Landolt, A. U., \& Wolff, M. J. 2003, ApJ, 594, 279

\section{REFERENCES}

Hirano, N., et al. 2004, ApJ, 616, L43

Knapp, G. R., Pourbaix, D., Platais, I., \& Jorissen, A. 2003, A\&A, 403, 993 Morrissey, P., et al. 2005, ApJ, 619, L7

Reimers, D., \& Cassatella, A. 1985, ApJ, 297, 275

Rich, R. M. 2005, Multiwavelength Mapping of Galaxy Formation and Evolution, ed. A. Renzini \& R. Bender (New York: Springer), 203

Sahai, R., Morris, M., Knapp, G. R., Young, K., \& 2003, Nature, 426, 261

Sahai, R., Morris, M., Sánchez Contreras, C., \& Claussen, M. 2007, AJ, 134, 2200

Sahai, R., \& Trauger, J. T. 1998, AJ, 116, 1357

Sahai, R., \& Wannier, P. G. 1988, A\&A, 201, L9

Schwarz, H. E., Corradi, R. L. M., \& Melnick, J. 1992, A\&AS, 96, 23

Soker, N. 1998, ApJ, 496, 833

Soker, N., \& Rappaport, S. 2001, ApJ, 557, 256

Sorensen, P. M., \& Pollacco, D. L. 2003, in ASP Conf. Ser. 303, ed. R. L. M.

Corradi, R. Mikolajewska, \& T. J. Mahoney (San Francisco: ASP), 494

van Winckel, H., Waelkens, C., Fernie, J. D., \& Waters, L. B. F. M. 1999, A\&A, 343, 202

Whittet, D. C. B. 1992, Dust in the Galactic Environment (Bristol: IOP), 67

Wood, B. E., \& Karovska, M. 2004, ApJ, 601, 502 2006, ApJ, 649, 410

Wood, B. E., Karovska, M., \& Hack, W. 2001, ApJ, 556, L51

Wood, B. E., Karovska, M., \& Raymond, J. C. 2002, ApJ, 575, 1057 